International Journal of Computers for Mathematical Learning (2006) 11:327-350

DOI: $10.1007 / \mathrm{s} 10758-006-0005-8$

(C) Springer 2006

CLAIRE CAZES, GHISLAINE GUEUDET,
MAGALI HERSANT and FABRICE VANDEBROUCK

\title{
USING E-EXERCISE BASES IN MATHEMATICS: CASE STUDIES AT UNIVERSITY
}

\begin{abstract}
E-Exercise Bases (EEB) are now used in the teaching of mathematics, especially at university. We discuss here the consequences of their use on the students' activity during computer lab sessions. Results stem from observations of several teaching designs organised in different French universities with three e-exercise bases. The analysis focuses on new tasks and on specific solving strategies, which appear using these resources. Moreover, specific didactic contract clauses are studied.
\end{abstract}

KEY WORDS: analysis of tasks, didactic analysis, didactic contract, E-exercise base, milieu, students' activity, undergraduate mathematics

\section{INTRODUCTION}

The work presented concerns the use of a certain kind of online resources, termed here e-exercise bases, to teach and learn mathematics. These resources consist mainly of classified exercises and propose, in addition to these exercises, an associated environment for each of them that can include suggestions, corrections, explanations, tools for the resolution of the exercise, and score. In what follows, e-exercise base is shorted to EEB.

Drill and practice products are EEBs, but many of the latter offer much more than drill. Moreover, EEBs are now more and more often integrated into complex virtual learning environments. Investigating these resources belongs to a trend of research that goes from programmed learning to the general concept of technology rich learning environment (Bruillard, 1997). About the more specific issue of integrating online resources in the teaching of mathematics, most of the recent works seem to focus on the products, with either taxonomy or evaluation purposes. For example, recently, Engelbrecht and Harding (2005) propose a classification using a radar chart with six radials: dynamics and access, assessment, communication, content, 
richness, independence. Among their categories, the products we will study can be "exercises and quizzes", but also "full courses".

These taxonomies are relevant and useful to compare and evaluate existing courses or to design online resources. However, they appear insufficient to determine the way these resources are really used, as Crowe and Zand (2000) write: "what is undoubtly lacking is proper evaluation of use, for there is often a serious mismatch between what the teacher intends, and what the student actually does." (p.146). Moreover, as it is noticed in Lagrange et al. (2003), many research studies are devoted to questions raised by the use of computer algebra systems (Artigue, 2002), spreadsheets, dynamical geometry systems or more general open interactive environments, ${ }^{1}$ and few studies are specifically dedicated to the students' activity in EEBs'environments.

However, several factors indicate that the study of EEBs is likely to point out different phenomena from what happens, for example, with computer algebra system (CAS). The use of a CAS requires specific learning, and a teaching design organised by the teacher. Using an EEB does not present any major technical difficulty; many EEBs have originally been designed for private use by students themselves. Nevertheless the main difference probably resides in the fact that, most of the time, CASs intervene to help students solve a mathematical exercise given by the teacher. In our situations, exercises are proposed by EEBs themselves and each EEB also comprises a specific environment, which can be more or less helpful. An EEB is a didactical construct, integrating self-learning objectives.

Finally, EEBs are actually used in the teaching of mathematics, and thus deserve the attention of mathematics education researchers. We make the a priori hypothesis that EEBs have specific didactical consequences on students' mathematical behaviour (on teacher's behaviour too, but this issue will not be considered here). For example, in their survey of the use of technology in mathematics courses in England, Ruthven and Hennessy (2002) observe that working in class with EEBs facilitate "trial and improvement" strategies for students (in this case secondary school students) and they notice that students are able to work at their own pace. We want to underline the consequences of the presence of an EEB in the learning environment upon the students'effective activities. It leads us to study questions such as: "what is the effective mathematical students activity during an EEB session?" "What actually differs from a traditional session?" 
The next section explains the theoretical tools used. Section 3 is an overview of the main features of the EEBs experimented. The associated learning designs and the data collected are specified in Section 4. Finally, specific students' cases are studied in Section 5. We conclude by a general discussion in Section 6 .

\section{THEORETICAL FRAMEWORKS}

The introduction of an EEB in class is likely to affect two main aspects of the teaching-learning processes. The first concerns the mathematical tasks proposed, and actually done by students. The second is the didactical situation. We use thus two different (but strongly interconnected) frameworks presented in the following paragraphs.

\subsection{Analysis of tasks and activities}

We refer to Robert (1998) and consider two dimensions of an assigned task: the status of knowledge and the level of knowledge actually needed for the task. The status of knowledge corresponds to the degree of innovation: the knowledge can be new, old, or can correspond to learning in progress. The level is linked to the autonomy required in students'activity for solving the task. Robert (1998) defines three levels of knowledge actually needed.

- The most elementary level and the corresponding exercises, termed direct applications corresponds to direct applications of an explicit knowledge in an isolated task.

- When students themselves have to recognise the need for a non-explicitly mentioned knowledge, the level is termed available.

- The intermediate level is termed by the French word mobilisable. It occurs when for example an adaptation is explicitly required. Many kinds of adaptations can intervene: the need to recognise that a given result applies, to find an appropriate method, to develop several steps in one reasoning and to establish relations. Adaptations are also required when there is a need to change point of view, to change setting (Douady, 1986) or register (Duval, 1995).

In her analysis of tasks, Robert also takes into account teacher's interventions. These interventions are indeed likely to affect the task 
originally proposed. Observations in traditional classes already revealed changes in the level of the task actually proposed due to teacher's interventions. The level of a task can shift this way from "available" to "mobilisable" or even "direct application" (Robert and Rogalski, 2005).

These categories of level defined by Robert must be carefully used, because they all depend on the student, on the status of associated knowledge, and on the available instruments. Here is a short, clear example. A student has to solve the equation: $x+\ln (x)=3$. It can be done with a graphic calculator, just after a course on similar uses of a graphic calculator. In such a case, the task will be a direct application. If the text of the exercise indicates: "study the variation of the function $f(x)=x+\ln x$ and use a dichotomy process", the level of task is mobilisable. Finally, the same task can be proposed without any indication. In such a case, the level of task is available and the student has to choose a method and recognize the appropriate knowledge. This example shows the importance to underline the context of the proposed tasks.

For the use of EEBs, this theoretical frame leads us to formulate the following questions: do EEBs sessions provide tasks of each level? Do they favor a specific level? Do "level shift" phenomena appear in sessions with EEBs? And in such cases, who is responsible for these phenomena: the teacher or the EEB? Are these phenomena similar to what happens in a paper and pencil session? Do students develop specific activities for solving some tasks with EEBs, different from what happens in a paper and pencil environment? Do new tasks appear in the work with an EEB?

\subsection{Analysis of the didactical situation}

Just like the teacher, an EEB can propose mathematical information, react to a student proposition etc. Thus produces thus modifications of the didactical situation, likely to affect the students' activity and learning processes. In order to analyse it, we refer to the theory of didactic situations (Brousseau, 1997) as a model for the construction and analysis of didactical situations. We use especially the notions of "milieu" and didactic contract.

EEBs do not propose "adidactical situations" with the meaning given to this term by Brousseau, leading students to construct new knowledge through the confrontation with an antagonistic milieu. EEBs propose on the contrary an environment designed to help 
students. The following features of an EEB will allow to describe the corresponding milieu : help, answer expected and feedback.

- Help are, for example, indications of method, explanations on a specific difficulty, short courses, hints or tools like an equation solver or a graphing tool. Students choose to use the helps or not.

- There are two kinds of answer expected by an EEB. The first one must be typed on the computer. Due to technical limitations, it is generally a numerical or algebraic answer, a multiple choice questionnaire, a zone to be selected on a graph etc. There is no analysis of a complex text written by a student. The second (kind of answer) must be produced on a paper.

- The EEB feedback depends on the kind of answer expected. When an answer is typed on the computer, most of the time, there is a simple analysis of this answer. The feedback says if it is right or wrong. Sometimes it gives a short explanation of a mistake or advice more or less adapted in the case of a wrong answer. This kind of feedback constitutes a retroaction; it can correspond to a validation or an evaluation. The EEB can attribute a mark, propose to solve a similar exercise and display a solution. It can also give a comment about the right answer. In the second case, when students write their answer on paper, the feedback is a detailed solution of the exercise. Students have to read and understand it, then compare it to their own solution.

After describing and analysing the milieu, it is necessary to specify the didactic contract associated with knowledge involved. The didactic contract is the set of rules between teacher and students which are specific to the knowledge taught. Bound by this didactic contract, students know they have to behave in a given situation by acting on it in specific ways. The situations involving an EEB are likely to induce modifications into the class' usual contract. The EEB integrates indeed its own contract, that we will term in what follows the EEB contract.

The didactical situations' framework leads us to study the following questions: is it possible to describe some contract's clauses of the EEB contract in the situations observed? How are students' responsibilities affected by the presence of the EEB? Does the EEB contract conflict with the teacher's contract, and in such cases, how is this conflict managed, and what are its consequences? 


\section{EXAMPLES OF E-EXERCISE BASES}

Presented here are the EEBs observed in the teachings observed. These resources are widely used in France, and they have very different features. They do not cover the whole range of possibilities for such products, but their differences allow us to avoid focusing on a specific product. For each of them, we first outline its main features and then focus on the didactic analysis of a specific exercise. Section 5 reports on observations of students working on these exercises.

\subsection{UeL (Online University)}

The UeL ("Université en ligne" which stands for "online university") is based mainly on learning objects developed through a partnership between French universities, available on the UeL web site (http://www.uel.education.fr). The set of learning objects is structured in modules. Each module contains different multimedia resources and is structured in three sections: course with hypertext and animations, exercises for drill and practice and tests for selfassessment. There are two types of exercises: interactive exercises with feedback and classical exercises where answers must be written on a paper and then compared with the solution proposed. For all exercises, a detailed solution is provided. This software does not provide $\log$ files.

Let us now see two very different types of exercises.

Interactive exercises are often «direct applications». Some of these are very simple and repetitive; they are not often proposed in paper and pencil sessions. Their interactive presentation on the EEB may make them more attractive to students. The following screenshot displays such an exercise (Figure 1).

This exercise belongs to a set of exercises corresponding to the same task. The students must decide whether or not Rolle's theorem ${ }^{2}$ applies to different functions. Similar exercises about the mean value theorem follow. In terms of task analysis, the level of task is "direct application": the theorem is explicitly mentioned in the text of the exercise. The status for the required knowledge is learning in progress.

In terms of environment analysis, the hints are comprised in the text itself. The hypotheses are indeed mentioned: students who answer negatively must find the condition which is not fulfilled by the function. The conclusion is also written on the screen: if students answer positively, they have to find a value of $c$ with $f^{\prime}(c)=0$. The 


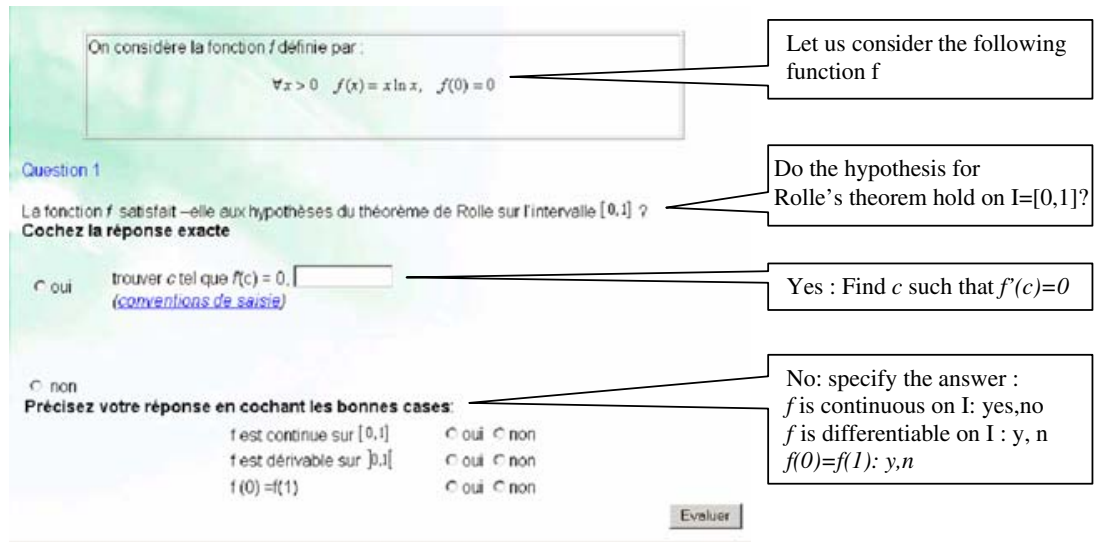

Figure 1. Exercise on Rolle's theorem in UeL.

expected answer is a multiple choice question MCQ with "yes"or "no", then a numerical value if the first answer was "yes", and a second MCQ if the answer was "no". The feedback analyses the students' answer. It gives additional justifications, and in the case of a positive answer the feedback points out the fact that several values of $c$ can exist in some cases. For the EEB, students must answer all the questions, otherwise the answer is not considered as valid: this is a clear aspect of the EEB contract.

The classical exercises can correspond to any of the three levels. We only give one example here (Figure 2).

The status of the knowledge involved is known as learning in progress. According to the EEB designers, the level of task seems to be direct application: "immediate understanding" is written in the left frame. However, the name of the corresponding theorem is not mentioned in the text of the exercise. Thus the level should be available. Students have to choose from a list comprising of three functions those admitting a reciprocal on $[-1 ; 2]$.

The environment of this exercise does not propose explicit hints. However, the students can access the text of the reciprocal functions theorem. There is no graphic help, but a graphing tool is available. The expected answer on the EEB is a MCQ with the name of the functions chosen. The EEB contract mentions here explicitly (on the previous screen) that a detailed written solution is expected on paper. The feedback of the EEB comprises a rough analysis of the MCQ answer: "you are right", or "you are wrong"; and a detailed solution which students have to read and compare to their own solution. 


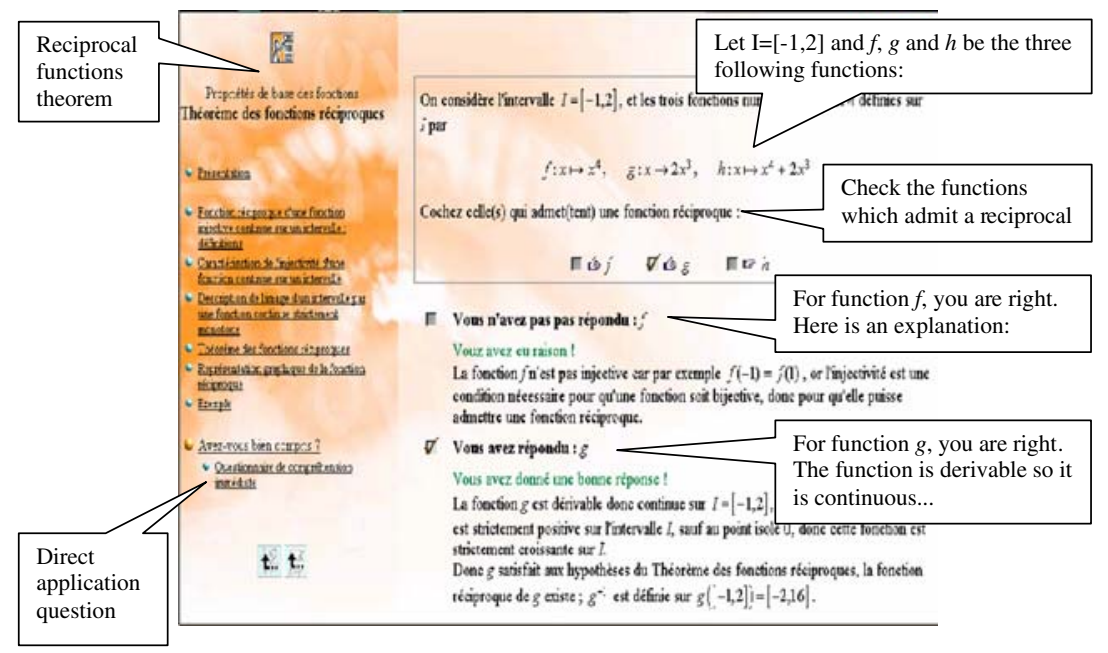

Figure 2. Reciprocal function exercise in UeL.

The environment can change the task's level if the students see that the name of the theorem is mentioned on the left frame. This text, designed to help the students to locate themselves on the EEB, can be turned into a mathematical help. Students can make a random answer, but it constitutes a breach of contract. The EEB contract asks for a detailed written answer, just like in paper and pencil sessions. It also requires the comparison of the written answer with the detailed solution proposed on the screen. This new task is explicitly mentioned. It can constitute a difficulty attached to the use of an EEB. Students may indeed not be prepared for this task. Moreover, they may not know whether or not they did it correctly if the teacher does not intervene. In standard sessions, the evaluation of students' work is done by the teacher who watches their individual work.

\subsection{WIMS}

WIMS (http://www.wims.auto.u-psud.fr) is a library of online interactive mathematical exercises that includes resources for all levels, from primary to tertiary education, formulated in six languages. Teachers can choose some of these exercises and elaborate their own online worksheets. Most of the exercises in WIMS require only a short answer: "Yes" or "No", an algebraic expression or a numerical value using an equation editor. The computer provides feedback to the student's answer and the correct answer using a computer algebra 
system, but no detailed solution. For each exercise, students get a mark. They can ask to do the same exercise several times, in order to improve their marks. In such a case, the structure of the newly proposed exercise remains the same, but the variables (numerical values, functions ...) differ. The software provides log files giving details of students' activities. Precise studies of these log files can be found in Vandebrouck and Cazes (2005).

WIMS comprises exercises corresponding to each of the three levels of tasks. We present now an exercise especially chosen because of its graphical feedback (Figure 3).

The exercise deals with knowledge about continuity and differentiability of functions at an available level of the task. Students have (indeed) to recognise that the given functions are of class $\mathrm{C}^{1}$. Then it is enough to compute the limits and the derivatives of the two given restrictions and to equal the results. There are no specific suggestions for that exercise. The expected answers are the numerical values $a_{1}$ and $a_{2}$. When students give an answer, the computer provides the following type of feedback:

The feedback is in the graphical register, while the text of the exercise is in the analytic register. Thus the level of the task does not seem to be modified by the feedback, but the milieu is enriched with a new register. This feedback does not necessarily help students to find the solution; it provides another viewpoint that helps only if students have sufficient knowledge to connect both registers (to make, for example, links between the derivative and the tangent's slope). For this exercise, a random answer, or several successive attempts is unlikely to lead to success without referring to the appropriate knowledge. In this case, it is not possible for students to develop a trial and errors strategy, because here the EEB does not permit several attempts on the same text: the functions and the numerical values

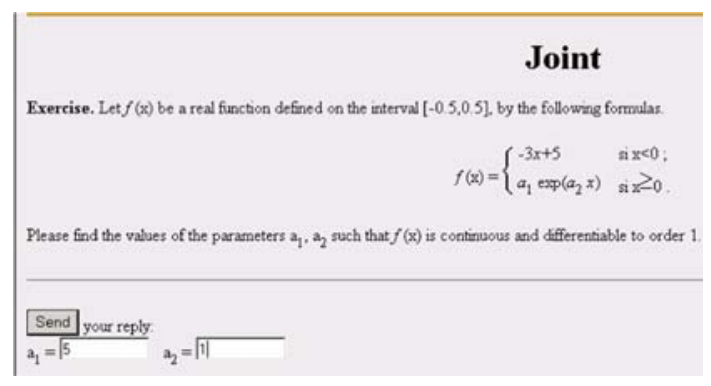

Figure 3. Joint exercise in WIMS. 


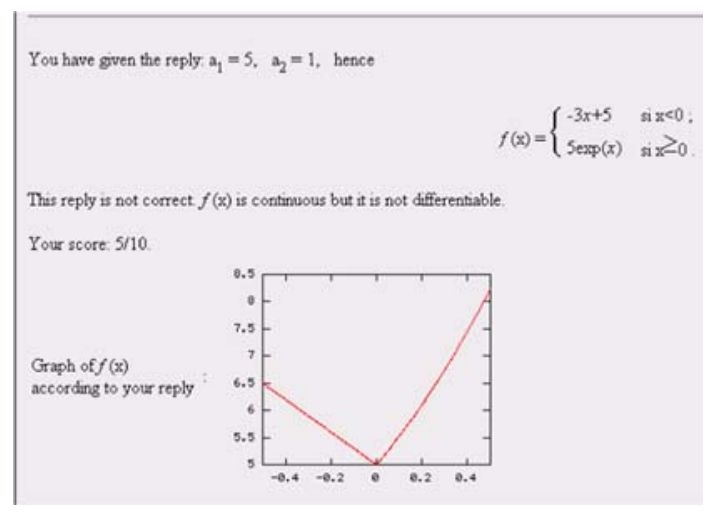

Figure 4. WIMS' feedback for the joint exercise.

change when students restart. In several other similar cases, this solving strategy is possible. For example, student may have ten attempts to get the correct result (Figure 4).

The explicit clause of the EEB contract is to find the values of the two parameters $a_{1}$ and $a_{2}$. There is also an implicit demand: using the feedback in case of a wrong answer to find a correct strategy. In this example, this task is very difficult. Students are not explicitly taught what they are supposed to do it. They face it alone without any associated advice and it is an entirely new task: in a standard class, teachers can naturally not produce graphs adapted to students' propositions.

\subsection{Braise}

BRAISE (http://www.tdmath.univ-rennes1.fr/braise.cgi) is an EEB for undergraduate students. Students obtain the exercises by choosing key-words: theme, type of task, and level of the exercise. The result of the choice is a list of exercise titles. Students choose one title; the corresponding exercise text is then displayed on the screen. Then students are supposed to solve the exercise with their paper and pencil. They can use the environment of the exercise, which comprises several kinds of suggestions, a complete solution of the exercise and one or two important ideas to be retained after solving the exercise. Four kinds of suggestions are available: short courses and presentation of methods (these two kinds are general, the same texts are associated with several exercises); graphic help, and indications (these suggestions are specific to one precise exercise). BRAISE provides log 
files with the list of all the windows opened by the students and the corresponding time. More details can be found (for example) in Gueudet and Houdebine (2003).

BRAISE is the less interactive software we used: there is no answer to be typed on the computer, thus no interpretation of the student's answer. However it can constitute a rich resource for the students, as the following example shows (Figure 5).

In BRAISE, the text of that exercise can be obtained by choosing one or several of the following key-words:

- Themes: extracted sequences, sequences $u_{n+1}=f\left(u_{n}\right)$, limit point, first exercises

- Level: easy

- Type of task: study the convergence.

The associated knowledge can have a different status depending on the associated learning design: a learning in progress, but also new knowledge (about methods to study the convergence of such sequences). The level of task is available, because students have to discuss the convergence of that sequence according to the value of its first term with no indicated method.

As for any other exercise in BRAISE, the environment comprises several kinds of suggestions, the solution and an idea that can be retained after doing the exercise ("Whatever the sequence, before starting complicated calculations, compute a few terms to have an idea of its nature."). There are four kinds of suggestions for that exercise:

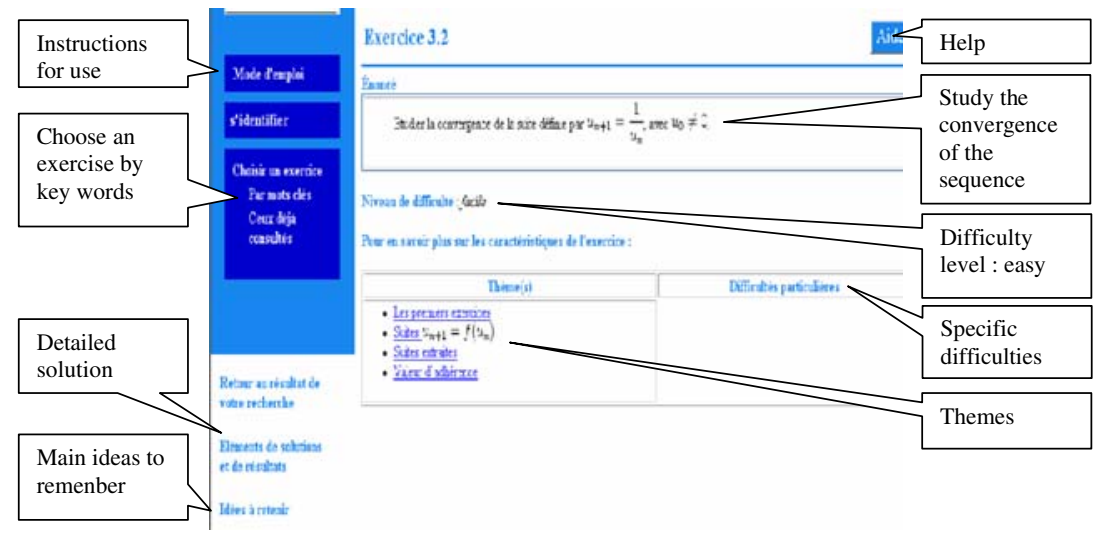

Figure 5. Sequences exercise in BRAISE. 
- Short courses about subsequences and limit points;

- Graphic advice, consisting of a drawing displaying the graph of the function $f(x)=1 / x$, the straight line $y=x$ and the first terms of $\left(u_{n}\right)$ for a given value of $u_{0}$, with the comment " $u_{0}=u_{2}$ ";

- A short text describing the method: "examining odd and even ranks";

- An indication: "Compute or draw $u_{1}, u_{2}, u_{3}$, according to $u_{0}$.".

Students have to search for the solution on paper, then the feedback consists of a detailed solution that they can freely access. There are almost no explicit constraints in the EEB contract for BRAISE. All the suggestions and the full solution are freely available. The intentions of BRAISE designers are described in a file entitled "instructions for use". These instructions present BRAISE features, and provide advice for its use, namely: "take a paper and pencil, you will work this way"; "search by yourself before displaying any help"; "try the help before the solution" and "write down the important ideas of the exercise". The corresponding file always appears in the left frame, but it has to be downloaded (students should print it the first time they use BRAISE, and then keep it with them). Moreover, there is no instruction about writing a detailed solution. The task which consists of reading the solution carefully and comparing it with their own solution remains an implicit clause of the contract.

In this example, it clearly appears that the suggestions change the task level. More precisely, they lower it from "available" to "mobilisable". Indeed, they indicate a method, and give hints for the starting point (noticing that the sequence can not be convergent). But the work left to the student is not a mere direct application. The milieu is enriched by the various suggestions; similar suggestions could be given by the teacher, but a teacher would probably give at most, one or two kinds of suggestions.

These presentations already show some EEB features in terms of task level offered and the EEB contract drawn up. As seen, EEBs may provide exercises of different levels. However, Braise only delivers exercises on the available or mobilisable level. In some cases, it is possible to anticipate level shift due to the hints (BRAISE) or to the indications given by the EEB's environment (UeL, Reciprocal function, the left frame). The EEB contract is more or less explicit. In all cases, students are supposed to search exercises with paper and pencil. In case of hints, they are asked to work seriously before 
consulting them. If the answer must be typed on the computer, it must have the right format (for example, in WIMS, joint exercise, $a_{i}$ must be a decimal number). Students are supposed to benefit from the feedback, if available, to improve their answer. Finally, they are asked to compare their solution with the EEB's one; as already mentioned this is a new difficult task.

Before studying how students deal with this new task, how EEB contract and teacher contract coexist in a class and more generally how EEB works in a real situation, it is necessary to present the context in which resources were used and the data collected.

\section{CASE STUDIES AND METHODOLOGY}

\subsection{Experimental Teachings}

Each case study involves one of the EEBs described in the preceding part, associated with a given teaching design.

The first teaching observed used UeL, at Paris 6 University, within an experimental teaching design for undergraduates. It is blended teaching: $30 \%$ distance learning and $70 \%$ presence learning integrating computer sessions. We observed seven training sessions (10 hours) with 30 students working on computers and a teacher present in the classroom. Thus all of these sessions correspond to learning in progress. This teaching was followed by a traditional paper and pencil assessment.

WIMS was used at the University of Evry, in two tutorials for first year students about calculus. It consisted of about 8 training sessions (11 hours) with 37 and 24 students. The students worked in pairs during the first tutorial and alone for the second one. This teaching was followed by a specific designed assessment. Half of the final mark was obtained on WIMS, in a computer assessment. The other half was obtained in a paper assessment but with exercises extracted from WIMS.

The last teaching used BRAISE at Rennes 1 University with firstyear students, $15 \%$ of the tutorials used BRAISE. We observed 6 sessions ( 9 hours): three were dedicated to introductory activities, and three were training sessions. Around 20 students attended these sessions with their usual tutorial teacher. A traditional assessment followed. 


\subsection{Data Collected}

In the experimentations related here, we tracked the students' behaviour in two ways: direct observations (UeL and BRAISE) and study of $\log$ files (WIMS and BRAISE).

\subsubsection{Direct Observations}

We carried out direct observations during the computer lab sessions. The general objectives of these observations were the following:

- see how the students manage their activity with the software (read the screen, stop, go back, search for course on the software, use paper, discuss with a neighbour);

- understand the mathematical activity of the students under these conditions (how they insert their reflection into what the software proposes: new exercises, corrections).

These observations supply us with a story of every session.

\subsubsection{Log files Observations}

Two software (BRAISE and WIMS) supply log files. The log files provide the connection time on each possible window (indications, courses, graphic help, solution) and for WIMS there are also global data with the average mark of one class of students on an exercise. The analysis of these data gives many details about the students' activity.

\section{STUDENTS' ACTIVITY DURING EEB SESSIONS}

This section is an analysis of students' activity working on exercises presented in Section 3. We focus first on exercises at the level of mobilisable or available, with individual student's cases. The observations of "direct application" exercises (as the first one about Rolle's theorem) are delivered at the end of the section with more general results.

\section{1. "Reciprocal Function" Exercise (UeL)}

\subsubsection{Marius' Activity: Presentation}

Marius writes the text of the exercise on his notebook. He uses his graphic calculator to draw the graph of each function. He copies these graphs to his notebook and deduces, by observing the graphs, 
whether the function has a reciprocal or not. His answer is right. Then the teacher says to the whole class: "you have to use the theorem of reciprocal functions". Marius reads the solution on the computer. He realises his answers are correct, but other arguments are used. For function $f$, the computer says it is an even function, thus $f$ can not be a bijection on $I$. For $g$, it computes the derivative. Marius does not read the last part of the solution. He takes his notebook and computes the derivative of $h$. He studies the sign of the derivative on $I$; it is strictly positive. Marius thus applies the theorem to deduce that $h$ admits a reciprocal function on $I$. The teacher goes past Marius when he starts the study for $h$ and says: "yes, this is the idea, compute the derivative."

\subsubsection{Marius' Activity: Analysis}

As mentioned in Section 3, the level of the task here is "available" and not "direct application" as assumed by the designers. The strategy presented in the computer's solution and the advice of the teacher, here coincide; it eventually leads the student to use this strategy. The level of the exercise is lowered to "direct application" by these advices, because the theorem is now explicitly mentioned and no further adaptation is required. This level's shift is similar to what is observed in standard sessions. It is due both to the computer and the teacher.

To solve the exercise, Marius first used his graphic calculator to obtain a graphical argument permitting him to decide whether the functions were bijections. This indicates that Marius has a good concept-image (Tall and Vinner, 1981) of bijection; it may be a consequence of the course presented on UeL, which contains many representations. Marius then showed he knew the theorem of reciprocal functions and was able to use it. He knows, thanks to the feedback, that his graphical method leads to a correct result. Listening to the teacher and watching the screen of the computer, he understands which method is expected, and he is able to apply it. There is only one stage missing in this process: he never asks the teacher, and he does not seem to ask himself, about the correctness of his first method. He does not really compare his solution with the computer's one: he just retains the computer's solution. The comparison task is important in all contexts: with or without an EEB. Students should develop the corresponding ability. Working with an EEB can foster such a development, especially as the teacher does not 
have to expose solutions for the whole class, and thus can dedicate more time to the discussion of individual solutions.

\section{2. "Joint" Exercise (WIMS)}

\subsubsection{Charles' Activity: Presentation}

Charles' activity is studied through the log file. He works for $34 \mathrm{~min}$ on the exercise. He makes in fact four successive attempts. During his first attempt, he searches for 9 minutes and gets the score 5 out of 10 . This means he only found the first missing value $a_{1}$. He quickly restarts the exercise. Then he works during 13 minutes and reaches the score 10 out of 10 . He restarts then the exercise two additional times, working 5 minutes each time, and obtaining the score of 10. Each time, he looks at the feedback, but only for a few seconds.

\subsubsection{Charles' Activity: Analysis}

Charles succeeds during his second attempt, thus 23 minutes after starting the exercise: it is quite a long time for such an exercise. The time spent in research is very important: 9 minutes, then 13 minutes, and two times 5 minutes. A similar exercise was proposed in the assessment at the end of the semester. Here is the corresponding extract of his sheet (Figure 6).

Charles develops the expected reasoning for $a_{1}$. He computes the right-hand and left-hand limits for each function and equates them. However, for $a_{2}$, he computes the increment ratio. There is a gap between what is expected and Charles' production. He did not resort to the most adapted knowledge, and thus did not use the most efficient technique. It explains the (long) time he spends on each exercise. The feedback and the restart's exercise, are not enough to make the expert knowledge available. Moreover, using the increment ratio actually works and there is no limitation of time for that exercise. As anticipated in Section 3, the feedback does not change the task's level. Adapted to the student's answer, the feedback is likely to help connecting the graphical and the analytic registers. It can contribute to the elaboration by the student of a continuity and differentiability concept-image. However, Charles does not stay enough time on the feedbacks and this elaboration cannot be observed.

Eventually, nobody examined Charles' personal strategy during the computer sessions; and he never received any advice on the most efficient method by the teacher during or after his work. As observed 
Exercise. Let $f(\mathrm{x})$ be a real function defined on the interval $[-0,5,0.5]$, by the following formulas.

$$
\begin{aligned}
& f(x)=-5 \operatorname{cxp}(-5 x) \operatorname{si} x<0 \\
& f(x)=a_{1}+a_{2} x \text { si } x \geq 0
\end{aligned}
$$

Please find the values of the parameters $\mathrm{a}_{1}, \mathrm{a}_{2}$ such that $f(\mathrm{x})$ is continuous and differentiable to order 1 .

$$
\begin{aligned}
& a_{1}=-5 \quad a_{2}=85 \\
& \lim _{x \rightarrow 0^{-}}-5 e^{-5 x}=-5 \text { dre calcul } \\
& \frac{-5 e^{-5 x}+5}{x-0}=\frac{-5\left(e^{-5 x}-1\right)}{x}=\frac{25\left(e^{-5 x}-1\right)}{-5 x} \text { or } a_{1}+a_{2} x=-5 \Rightarrow e_{1}^{-5 x}--5 \\
& \frac{a_{1}+a_{2} x-a_{1}}{x}=a_{2} \Rightarrow 1
\end{aligned}
$$

Figure 6. Charles' sheet.

in Marius' case, a correct answer does not mean the method is correct or is the best one. Teachers and students must be aware of such EEB pitfalls.

\section{3. "Sequences" Exercise (BRAISE)}

\subsubsection{Fanny and Camille's Activity: Description}

Fanny and Camille's case is described by direct observations and log files. Fanny and Camille, after reading the text of the exercise, start searching together the solution on a paper. They try to apply the general method: looking for $l$ with $l=1 / l$. They find $l=1$ or $l=-1$, but they have no idea about what to do afterwards. So they agree on opening the hints, 3 minutes and 16 seconds after opening the exercise.

The first help they open is a short course about limit points. They read it carefully (during 2 minutes and 25 seconds). They do not write anything on their paper, and do not understand how to use it for that exercise; they never came across that notion before. Then they turn to the graphical help, but they only have a quick look at it (11 seconds), and do not seem to notice the comment " $u_{0}=u_{2}$ ". They turn to the advice "Compute or draw $u_{1}, u_{2}, u_{3}$ according to $u_{0}$ ". They 
follow that advice, and compute together on one paper $u_{1}=1 / u_{0}$, $u_{2}=u_{0}, u_{3}=1 / u_{0}$. Fanny (who is doing the writing) is the first to understand what is happening, she says to Camille: "it cannot be convergent, because one term is always $u_{0}$, and the other $1 / u_{0}$ ". They write "divergent" on their paper, and do not examine the cases $u_{0}=1$ or $u_{0}=-1$. They never write a detailed proof; they stop once they are both convinced.

After that Fanny and Camille do not turn immediately to another exercise. They notice that they did not try the kind of help called "method". So they use that link and read the method "examine odd and even ranks". They read it carefully, for about 2 minutes, and realise that they applied themselves such a method; but they do not write it down. Then they turn to the solution and read it; they observe that in their own solution, they neglected the cases $u_{0}=1$ and $u_{0}=-1$, mentioned in the solution.

\subsubsection{Fanny and Camille's Activity: Analysis}

The first point we want to emphasise is that their personal search is very short: around 3 minutes. It means that these students used the help just after having encountered the first difficulty. It breaks the EEB contract as expressed by the designers of BRAISE in the instructions for use, and recalled by the teacher at the beginning of the session: make all possible attempts before opening the help. Is it really different from what happens in usual tutorial sessions? In such a session, the students would probably call the teacher at the same moment. But the teacher can refuse to give a suggestion so early.

Secondly, it appears that Fanny and Camille opened many windows, looking for the most adapted help. The variety of possible helps is a richness, but also a pitfall of BRAISE, as said in Section 3.3, when it lowers the level of the task. In a classical tutorial, the teacher would probably propose only one advice. It is less likely to reduce students' mathematical activity. However, most of the time, in class, the suggestions are formulated for the whole class. Thus they can be unsuitable, or can be formulated at the wrong moment.

Finally, it is important to take into account that Fanny and Camille make a careful reading of the texts on the screen. This task could exist during a standard tutorial, if they start for example reading their course. During the observations, it seemed however more frequent in a session with BRAISE. Many students seem eager to collect lots of information to benefit from the enriched milieu. For this exercise, the solution was very short. But for more complicated 
problems, sometimes reading the solution, and comparing it with the one they found is for the students an activity they practice very seriously. Fanny and Camille even read the "examine odd and even ranks" method after finding the solution. It turned out to be really useful, because they reinvested this method in the homework they had to hand in the following session: studying the sequence defined by $z_{n+1}=\sqrt{ } 2-z_{n}$ according to $z_{0}$.

\subsection{General Observations Results}

The main general result is the importance of the students' engagement in their work on EEB. In all experiments, students worked really hard during the sessions. Log files show that many of them even worked outside the sessions to complete their exercises. Of course, for the WIMS experiment, the fact that the work on EEB is taken into account in the semester's mark may play an important part in students' engagement. However, we consider that implicit modifications with regards to a standard situation and especially the students' expectations from the teacher are also essential. Indeed, students know that they cannot just wait until the solution is given. They must at least start to look for a result because they can never be sure to receive a direct advice from the teacher.

The second point to notice is the variety of students' paths. Indeed, EEBs allow students to go back, to make several attempts and to ask for help if they need to. Students can also follow different paths among exercises. The studies of log files on WIMS experiment clearly display these different paths and paces. However, a pitfall of these possibilities of personal paths is the "scoring effect". It has been observed on WIMS log files. Some students keep on doing the same "direct application" exercises instead of facing more difficult exercises. They seem to aim at obtaining the maximal mark (10 over 10) for these exercises, even if they have to renew the same exercise many times. Series of 10 for the same exercise can be observed in the log files.

Finally, all the observations indicate that the work on direct applications exercises is very beneficial. For example, the observations show that exercise about Rolle's theorem prompts a good rereading of the course, which the students might not have done in depth at home by themselves. In such a case, what is expected coincides with what really happens, regarding effective students' activity and implicit contract's rules. Due to their technical, even repulsive aspect, some of these exercises might not even be organised 
by the teachers in traditional sessions and sent back implicitly to a personal work of the students. Let us assume that a critical quantity of exercises or examples of applications is necessary to learn a theorem, acquire a technical method, or constitute a stock of examples, this quantity is certainly more easily reached in a teaching design involving an EEB. Moreover, each student can reach his own necessary quantity.

\section{DISCUSSION AND CONCLUSION}

This part exposes and discusses the answers to the questions presented in Section 2. Two main aspects of the teaching-learning processes were distinguished: the tasks and activities and the didactical situation. These aspects are strongly interconnected; however, we retain the same distinction as for the results' presentation, with an emphasis on the didactical contracts (EEB and teacher's one) within the didactical situation.

\subsection{Tasks and Activities}

\subsubsection{Do EEB Sessions Provide Tasks of Each Level? Do They Favour a Specific Level?}

The a priori EEBs' analysis (Section 3) shows that EEBs may deliver all types of exercises. However, observations indicate EEB sessions are especially convenient for direct application exercises because there are no mismatches between the expected activity and the effective one. For upper level exercises, observations show difficulties to catch the best solving strategy (as in Charles' example) or to evaluate the student's strategy (for instance, Marius and his graphical strategy). Indeed, due to the fact that the EEB's analysis of answer is poor, this evaluation task is generally the responsibility of the student.

\subsubsection{Do "Level's Shift" Phenomena Appear in EEB Sessions?}

"Level shift" phenomena were observed during EEB sessions. These shifts can be due to the environment of the exercise. It appears clearly in the case of Fanny and Camille, who open all the possible suggestions, and follow advice. But shifts can also come from the teacher: in Marius' case, the teacher and the EEB simultaneously suggest the same method. The environment does not always affect the level: in Charles' case, the feedback does not lower the level. Thus we retain 
that, in some cases, the students' task is modified and the level is lowered from available to mobilisable or even direct application. As already said, these phenomena are not specific from EEBs. However, the situation seems worse with an EEB, because the teacher does not control the students' access to help or suggestions.

\subsubsection{Do Students Develop Specific Activities and Strategies with EEB?}

The changes induced by the presence of an EEB may concern the strategies employed by students. For example, Marius uses a graphical strategy for the "reciprocal function" exercise, probably because of the course presentation on UeL. In other cases that we did not detail here, we observed trial and errors strategies, for MCQ and numerical answers. That may be interesting to study but, sometimes, students use the feedback to guess the answer without actually making any mathematical sense of their proposition.

We propose to term "robust" tasks that cannot lead to such a shift in the actual activity, compared to the expected activity. With an EEB, the teacher must be especially aware of these possible shifts. And the EEB designers have to choose carefully the tasks they propose and to anticipate the student's strategy taking in account feedbacks. The proposed tasks must be robust enough to guarantee an actual activity corresponding to what is expected, and to minimise the level's shift. It could be done for example, as we saw it for the "Joint" exercise, by introducing feedback in another setting than the exercise's text.

\subsubsection{Do New Tasks Appear in the Work with an EEB?}

As already said, an important new task exists: the comparison of the students' and the EEB's solution. Students must first, carefully read the EEB solution and understand it (as Fanny and Camille did). Then, they have to observe the similarities and differences with their own answer. They should even decide which one is the most appropriate if both solutions obviously differ. They are probably convinced that the computer's solution is better; but they should examine their own solution to understand its potential disadvantages. This may be done (Fanny and Camille notice after reading the EEB solution the particular case of $u_{0}=1$ or $u_{0}=-1$ ), but not always (Marius's case). 


\subsection{Didactical Contracts and Their Consequences}

\subsubsection{Is It Possible to Describe Some EEB Contract's Clauses in the Situations Observed?}

The a priori analysis of the EEBs shows the existence of specific contract's clauses. Some of them (essentially the answer format) are resistant and students cannot free themselves from. Others are not and observations show how students may transgress them, as Fanny and Camille, searching only for a few minutes before consulting the hints. A important point is that some others clauses are too difficult to be kept by students. For instance, the students do not understand that the comparison between their solution and the EEB's one (Marius case) or that the consideration of the feedback (Charles' case) are expected, tasks. Moreover, the importance of students' engagement noticed in the observations may be a consequence of an implicit EEB contracts' clause. Indeed, students know that they have to act, otherwise nothing will happen.

\subsubsection{How are Students' Responsibilities Affected by the Presence of the EEB?}

Some new responsibilities clearly appear. A central new responsibility is the choice by students of their own path among the exercises. In standard sessions, the teacher is principally responsible for change in the activity and there are fewer possibilities of individual paths. In the EEB contract, more choices are offered to students. They have more responsibilities, which also means they have to produce a more difficult cognitive effort.

\subsubsection{Does the EEB Contract Conflict With the Teacher's Contract?}

In Marius' case, observations show an agreement between the two contracts. In some other cases, conflicts may appear. For example, EEBs may favour graphic strategies while the teacher may reject them. Difficulties may appear about written proof and justification expectations. For instance, WIMS contract does not ask students to write the whole solution which may conflict with the didactical contract established with the teacher which requires, in this case, students to keep notes. Moreover, it may prevent the teacher from knowing the students' procedures and difficulties. The example of exercise "Joint" is particularly significant with regard to this: in the EEB contract, a numerical answer from the student is sufficient and the teacher doesn't know how the student obtained his answer. 


\subsection{Perspectives}

No doubt this first EEB approach requires further investigations. However, we assume that the presentation sheds some light on possible learning-teaching processes with EEB sessions. For instance, it emphasises the importance of direct application exercises and the precautions to develop with upper level exercises. But this study mainly leads us to point out an often missing step in an EEB learning session. The actual students' strategies are not often examined and compared with what is expected. Even when the student's answers are correct, an EEB cannot give a precise analysis of his/her procedure. Moreover, some new important tasks implicitly devoted to the students are not well identified and bring new difficulties with them. The question of the institutionalisation and reorganisation of knowledge involved in EEB sessions appears then as a crucial point.

\section{NOTES}

${ }^{1}$ Like the connected curriculum project (Bookman and Malone, 2003).

2 Rolle's theorem : Let $f:[\alpha, \beta]$ - IR be a continuous function on $[a, b]$, differentiable on $(a, b)$, and such that $f(a)=f(b)$. Then, there exists a $c$ in $(a, b)$ such that $f^{\prime}(c)=0$.

\section{REFERENCES}

Artigue, M. (2002). Learning mathematics in CAS environment: the genesis of a reflection about instrumentation and the dialectics between technical and conceptual work. International Journal of Computers for Mathematics Learning 7: 245-274.

Bookman, J. and Malone, D. (2003). The nature of learning in Interactive technological environments. A proposal for a research agenda based on grounded theory, Research in Collegiate Mathematics Education V, American Mathematical Society.

Brousseau, G. (1997). Theory of didactical situations in mathematics: Didactique des mathématiques, 1970-1990. Dordrecht: Kluwer Academic Publishers.

Bruillard, E. (1997). Les machines à enseigner. Paris: Hermès.

Crowe, W.D. and Zand, H. (2000). Computers and undergraduate mathematics 3: Internet resources. Computers \& Education 35(2): 123-147.

Douady, R. (1986). Jeux de cadres et dialectique outil-objet, Recherches en Didactique des Mathématiques:7.2, 5-31, Ed. La Pensée Sauvage, Grenoble.

Duval, R. (1995). Sémiosis et pensée humaine: registres sémiotiques et apprentissages intellectuels, Ed. Peter Lang.

Engelbrecht, J. and Harding, A. (2005). Teaching undergrate mathematics on the internet. Part 1: Technologies and taxonomy. Educational Studies in Mathematics 58: $235-252$. 
Gueudet, G. and Houdebine, J. (2003). Une base d'exercices en ligne à l'université, Actes du colloque ITEM, IUFM de Reims. http://www.reims.iufm.fr/Recherche/ ereca/itemcom/.

Lagrange, J.-B., Artigue, M., Laborde, C. and Trouche, L. (2003). Technology and mathematics education: A multidimensional study of the evolution of research and innovation, Second international handbook of Mathematics Education (pp. 237269). Kluwer Academic Publishers.

Robert, A. (1998). Outils d'analyses des contenus mathématiques à enseigner au lycée et à l'université. Recherche en Didactique des Mathématiques 18(2): 139140.

Robert, A. and Rogalski, J. (2005). A cross-analysis of the mathematics teacher's activity. An example in a French 10th-grade class. Educational Studies in Mathematics 59: 269-298.

Ruthven, K. and Hennessy, S. (2002). A practitioner model of the use of computerbased tools and resources to support mathematics teaching and learning. Educational Studies in Mathematics 49(2-3): 47-86.

Tall, D and Vinner, S (1981). Concept image and concept definition in mathematics, with special reference to limits and continuity. Educational Studies in Mathematics 12: $151-169$.

Vandebrouck, F. and Cazes, C. (2005). Analyse de fichiers de traces d'étudiants: Aspects didactiques, Revue STICEF, Vol. 12, ISSN: 1764-7223, http://www.sticef.org.

\section{Claire Cazes}

Université Denis Diderot (Paris 7) DIDIREM

Boîte Courier 7018 2, Place Jussieu, 75251, Paris Cedex 05, France

E-mail: cazes@ccr.jussieu.fr

Ghislaine Gueudet

CREAD-IUFM de Bretagne Site de Rennes

153 rue de Saint-Malo, 35043, Rennes cedex, France

E-mail: ghislaine.gueudet@bretagne.iufm.fr

\section{Magali Hersant}

IUFM des Pays de Loire 4, chemin de Launay Violette

BP 12227, 44332, Nantes Cedex 3, France

E-mail:magali.hersant@paysdelaloire.iufm.fr

Fabrice Vandebrouck

Université Denis Diderot (Paris 7) DIDIREM

Boîte Courier 7018 2, Place Jussieu, 75251, Paris Cedex 05, France

E-mail: fabrice.vandebrouck@univ-evry.fr 